\title{
Wenn Sparmassnahmen Einsparungen verhindern
}

\author{
Josef E. Brandenberg \\ Dr. med., Facharzt Orthopädie und Traumatologie, Präsident FMCH
}

Wie ein Mantra wird seit Jahren der medizinische Fortschritt als eine Hauptursache für Kostensteigerungen gebrandmarkt. Die Einsparungen durch Innovation werden ausgeblendet. Tatsächlich gibt es jedoch zahlreiche Beispiele dafür, wie der medizinische Fortschritt nicht nur die Behandlung und die Lebensqualität der Patienten verbessert hat, sondern auch zu Kostenersparnissen geführt hat.

\section{Wie neue Medikamente Magen- operationen obsolet machten}

Beim Staatsexamen 1976 mussten angehende Ärzte und Ärztinnen in den mündlichen und schriftlichen Prüfungen noch mehrere Fragen zur Vagotomie beantworten. Diese operative Behandlung des Magengeschwürs war damals noch nicht obsolet. Das «Magengeschwür», das Ulcus duodeni, war ein Volksleiden der angeblich gestressten Bevölkerung, die Vagotomie die Therapie der Wahl. Ein Jahr später war dieser Eingriff vom Operationsprogramm verschwunden, von Cimethidin (Tagamet $^{\circledR}$ ) verdrängt. Das Medikament war ein Riesenerfolg und wurde angeblich einer der ersten Blockbuster [1, 2] der Pharmageschichte. Von den noch viel grösseren Einsparungen durch den Wegfall der Vagotomie spricht niemand.

\section{Wie die moderne Überwachung von Geburten Behinderungen reduzierte}

Die Geburtshilfe erlebte in den 1970er Jahren einen enormen technischen Fortschritt. Dank klinischer Forschung wurde in Zusammenarbeit mit der medizintechnischen Industrie die apparative Überwachung des Geburtsvorganges mit Hilfe von Herztonwehenschreibern, mit fetalen Blutgasanalysen und Kopfschwarten-Elektroden entwickelt. Das frühe Erkennen von Sauerstoffmangel führte zwar zu einem Anstieg der Kaiserschnitte, gleichzeitig verschwanden aber auch durch den Geburtsvorgang bedingte Hirnschädigungen weitgehend. Dank der modernen Geburtsüberwachung sind vielen Kindern Spasmen und andere Behinderungen erspart geblieben und damit auch lebenslange Beeinträchtigungen und Belastungen für die betroffenen Familien. Das Unterlassen der Über- wachung gilt heute als Verletzung der Sorgfaltspflicht, wie der jüngste - für alle Beteiligten - tragische Fall zeigt [3]. Die gerichtlich verfügte Entschädigung deckt bei Weitem nicht die Folgekosten für medizinische Behandlungen, für Hilfsmittel, für Sonderschulung und für lebenslange IV-Renten, lässt aber erahnen, was moderne Geburtshilfe bislang an Kosten eingespart hat.

\section{Wie die klinische Forschung Kindern Operationen ersparte}

Ende der 1970er Jahre waren in verschiedenen Kliniken noch ganze Abteilungen mit Kindern belegt, vor allem Mädchen, denen vor der Pubertät wegen Einwärtsgang die Hüften korrigiert wurden. Der stärker nach vorne abgewinkelte Schenkelhals wurde korrigiert - indem im Rahmen einer intertrochantären Osteotomie der Knochen durchtrennt und rotiert wurde - zuerst auf der einen Seite, nach Ausheilung auf der Gegenseite. Klinische Studien - als Beispiel sei die hervorragende Dissertation von Schwarzenbach [4] zitiert - zeigten, dass sich die stärker abgewinkelte Stellung des Oberschenkelhalsknochens bis zum Wachstumsabschluss spontan korrigiert und die operative Behandlung keine Vorteile bringt [5]. Die Derotations-Osteotomie ist innert kürzester Zeit vom Operationsprogramm verschwunden. Wiederum hat die klinische Forschung zu gewaltigen Einsparungen geführt.

\section{Weitere Forschungserfolge: Stabilschuh statt Bandnaht}

Die Distorsion des oberen Sprunggelenkes ist eine der häufigsten Verletzungen. Lange galt die Lehrmeinung, die gerissenen Bänder müssten genäht werden, bis 
1987 eine prospektive randomisierte Studie [6] zeigte, dass die konservative Behandlung gleich gute Resultate wie die Bandnaht bringt. In einer weiteren Studie konnte sogar die Überlegenheit der funktionellen Behandlung, also ohne starre Ruhigstellung, gegenüber der Operation nachgewiesen werden [7]. Seither machen die Hersteller und Verkaufsstellen von Stabilschuhen das grosse Geschäft. Die Naht der frischen Bandrupturen des Sprunggelenks ist vom Operationsprogramm verschwunden. Ein weiteres Beispiel für Einsparungen dank klinischer Forschung!

\section{Hüftsonographie-Screening - eine Erfolgs- geschichte trotz administrativer Hürden}

1980 publizierte der österreichische Orthopäde Reinhold Graf [8] über die heute als Hüftultraschall allgemein bekannte Sonographie der Säuglingshüften. Seither haben unzählige Studien bewiesen, dass das sonographische Screening gut geeignet ist, die Hüftdysplasie als häufigste Fehlbildung bei etwa vier Prozent aller Neugeborenen zu erkennen und damit eine frühzeitige Behandlung zu ermöglichen. So werden die schwerwiegenden Folgen der Hüftdysplasie - lange Spitalaufenthalte der Kleinkinder, mehrere Folgeoperationen, Entstehung von vorzeitiger Arthrose - vermieden. Dank beherzten und beharrlichen Kinderorthopäden und Kinderchirurgen - z.B. Fritz Hefti in Basel, Ulrich Exner in Zürich, Martin Schilt in Luzern [9] - hat sich die Sonographie der Säuglingshüfte auch in der Schweiz etabliert und damit nicht nur viel Leid erspart, sondern auch grosse volkswirtschaftliche Einsparungen ermöglicht. Unglaublich aber wahr, dass diese Untersuchung im Jahr 2004 aufgrund der fehlgeleiteten Sparhysterie des Departements des Innern beinahe aus dem Grundleistungskatalog gestrichen worden wäre und nur durch Proteste der Ärzteschaft und Unterstützung der Nationalen Ethikkommission [10] erhalten blieb. Heute beobachtet man die schweren Folgen von Hüftdysplasien fast ausschliesslich bei zugewanderten Menschen, nicht selten aus Ländern, deren Gesundheitswesen angeblich dem der Schweiz überlegen sein sollen.

\section{Forschung reduziert Krankheiten und entlarvt ungeeignete Behandlungs- methoden - muss aber finanziert werden}

Tr.

E. Brandenberg

Facharzt Orthopädie und

Traumatologie

Präsident FMCH

Rebstockhalde 18

CH-6006 Luzern

josef.brandenberg[at]hin.ch zum Verschwinden bringen und damit zu gewaltigen Einsparungen führen. Im Vergleich dazu fallen die Ergebnisse der von Ökonomie und Politik inszenierten Sparübungen sehr bescheiden aus.

Die Finanzierung von Forschung und Entwicklung wird jedoch zunehmend schwieriger. An einem gesundheitspolitischen Podium der Arbeitsgemeinschaft für Endoprothetik vom Dezember 2016 in Berlin klagten mehrere Chefärzte von Unikliniken, in Deutschland seien klinische Studien mangels finanzieller und personeller Ressourcen kaum mehr möglich. Und auch in der Schweiz zeichnen sich vergleichbare Probleme ab. Mit dem Krankenversicherungsgesetz KVG wurden 1996 flächendeckend die WZW-Kriterien eingeführt. Leistungen wie medizinische Behandlungen oder Medikamente werden von der obligatorischen Krankenpflegeversicherung OKP erst dann vergütet, wenn ihre Wirksamkeit, Zweckmässigkeit und Wirtschaftlichkeit nachgewiesen sind. Die Kosten für Forschung und Entwicklung - wie übrigens auch die Kosten für die Weiterbildung - sind ausdrücklich von der Leistungspflicht der Sozialversicherungen ausgeschlossen. Aber wer soll diese bezahlen?

Mit der Einführung der Fallpauschalen in der OKP werden die Spitäler zusätzlich zum Sparen gezwungen. Für klinische Forschung und Entwicklung bleiben nur noch Erträge aus Zusatzversicherungen und die Anwerbung von Drittmitteln. Doch die Zahl der Zusatzversicherten nimmt stetig ab, und die Zusatzentgelte stehen zunehmend unter Druck der Regulationsbehörden. Nicht zuletzt werden auch Zuwendungen der Industrie an Spitäler und Ärzteschaft gerne einmal an den Pranger gestellt und bergen damit ein gewisses Reputationsrisiko für ein forschendes Spital.

\section{Fazit und Zusammenfassung}

Die hier aufgeführten Beispiele der Magenoperationen, der Geburtsüberwachung, der Osteotomien, der Sprunggelenksverletzungen und der Hüftsonographien zeigen, dass der medizinische Fortschritt in der Vergangenheit nicht nur Kosten erhöht, sondern auch Einsparungen ermöglicht - und Patienten und ihren Angehörigen viel Leiden und verzichtbare Behandlungen erspart hat. Die unverzichtbare Basis für Fortschritte wie diese sind Erkenntnisse, die aus klinischer Forschung gewonnen werden. Leider geraten diese Forschungstätigkeiten unter zunehmenden finanziellen Druck. Sparmassnahmen zulasten klinischer Forschung führen jedoch zunehmend zur Innovationsblockade, verhindern paradoxerweise echte Einsparungen und machen das Gesundheitswesen nicht einmal billiger, sondern ausschliesslich schlechter. 


\section{Literatur}

1 https://de.wikipedia.org/wiki/Cimetidin

2 https://de.wikipedia.org/wiki/Blockbuster

3 Tagesanzeiger vom 28.10.2017. „Zürcher Spital muss für behindertes Kind Genugtuung zahlen“. Bericht über das Urteil des Zürcher Obergerichts.

4 Schwarzenbach U (1971). Die Rückbildungstendenz der idiopathisch vermehrten Antetorsion des Schenkelhalses. Inaugural-Dissertation. Medizinische Fakultät, Universität Zürich. Arch Orthop Unfallchir;70:230-42

5 Jani L, Schwarzenbach U, Afifi K, Scholder P, Gisler P (1979). Verlauf der idiopathischen Coxa antetorta. Kontrolluntersuchungen von 148 Patienten bei Wachstumsabschluss. Orthopäde;8:5-11.
6 Korkala O, Rusanen M, Jokipii, Kytömaa J, Avikainen V (1987). A prospektive study of the treatment of severe tears of the lateral ligament of the ankle. Int Orthop;11(1):13-7.

7 Kaikkonen A, Kannus P, Järvinen M (1996).Surgery versus functional treatment in ankle ligament tears. A prospective study. Clin Orthop Relat Res. 1996 May;(326):194-202

8 Graf R (1980). The diagnosis of congenital hip-joint dislocation by the ultrasonic Combound treatment. Arch Orthop Trauma Surg;97(2):117-33.

9 In memoriam Martin Schilt. Schweiz Ärzteztg. 2012;93(11):405

10 Sonographisches Hüftscreening bei Neugeborenen nach Graf Schweiz Ärzteztg. 2004;85(12):620 